\title{
Personal audio devices and the risk of hearing loss in young adults
}

\author{
Salwa Mourad Abd El-Mawgoud
}

Assistant Professor of Audio-vestibular Medicine, Audio-vestibular Unit, E.N.T. Department, Faculty of Medicine, Sohag University, Sohag, Egypt

\section{Type of article: Original}

\begin{abstract}
Background: Personal listening devices (PLDs) are considered one of the most popular sources of recreational noise that may result later on in hearing loss. However, most PLD users are unaware that they are putting themselves at risk of sensorineural hearing loss.

Objective: to assess and early discover subtle changes in hearing sensitivity caused by personal audio devices (PADs).

Methods: In this case control study, the participants were selected through simple random sampling with age range from 14 to 20 years. The study was conducted at the Audiology Unit, Sohag University Hospital, Sohag, Egypt between March 2018 and January 2019. The participants were divided into two groups: Group I (case group) with history of regular use of PADs and Group II (control group) who never use PADs. All participants underwent pure tone audiometry (PTA), extended high frequency (EHF) audiometry, immitencemetry and transient evoked otoacoustic emissions (TEAOE). Data were analyzed using IBM-SPSS version 23. Statistical methods used include descriptive analysis (mean, range and standard deviation), odds ratio and Pearson correlation test.

Results: The average duration of PAD use in the case group was 2 years with $68.29 \%$ of PLD use $\geq 4$ days/ week, $63.41 \%$ of PLD use $\geq$ one hour daily and $39.02 \%$ preferring high volume. Tinnitus was the most common problem that occurred immediately after PAD use, followed by hearing loss. The EHF results showed a highly statistically significant difference in the threshold between the two groups at all tested frequencies (the odds ratio was 165.00 with a high degree of significance $p=0.0004$ ). The whole case group showed hearing threshold shift at all frequencies from 10 to $16 \mathrm{KHz}$ at both ears. The largest hearing threshold shift occurred in the case group that used PADs for two or more years. The odds ratio for Transient Evoked Otoacoustic Emissions (TEOAE) response was 1.0649 with no statistically significant differences between case and control groups $(\mathrm{p}=0.8264)$. However, the mean amplitude of the overall TEOAE spectrum was lower in the case group compared with the controls. In the right ear, it was $16.85 \pm 3.02 \mathrm{~dB}$ in the case versus $18.92 \pm 3.14 \mathrm{~dB}$ in the control with the degree of the significance less than 0.01 . In the left ear, it was $16.92 \pm 4.28 \mathrm{~dB}$ in the case versus $19.01 \pm 3.76$ in the controls with the degree of the significance less than 0.05 .
\end{abstract}

Conclusion: The current study suggests that the long-term use of PLDs can impair hearing function, so a screening protocol should be considered for early identification of NIHL in PLD users.

Keywords: Noise, hearing loss, personal audio devices

\section{Abbreviations / Acronyms:}

EHF: Extended high frequency; NIHL: Noise-induced hearing loss; OAE: Otoacoustic Emission; PAD: Personal audio device; PLD: Personal listening device; PTA: Pure tone audiometry; SNR: Signal-to-noise ratio; TEOAE: Transient Evoked Otoacoustic Emissions; WHO: World Health Organization

\section{Corresponding author:}

Assistant Professor Dr. Salwa Mourad Abd El-Mawgoud, Audio-vestibular Unit, E.N.T. Department, Faculty of Medicine, Sohag University, Sohag, Egypt. Tel: +966545851363, E-mail: salwa_mawgoud@yahoo.com

Received: November 10, 2019, Accepted: December 30, 2019, Published: March 2020

iThenticate screening: December 30, 2019, English editing: February 02, 2020, Quality control: February 16, 2020

This article has been reviewed / commented by three experts

Ethics approval: Ethical Committee of the Faculty of Medicine, Sohag University, Egypt (Ref.: 23/2019)

(C) 2020 The Authors. This is an open access article under the terms of the Creative Commons Attribution-NonCommercialNoDerivs License, which permits use and distribution in any medium, provided the original work is properly cited, the use is non-commercial and no modifications or adaptations are made. 


\section{Introduction}

Noise-induced hearing loss (NIHL) can result from exposure to occupational noise and non-occupational or recreational noise. One of the important sources of recreational noise is Personal listening devices (PLDs) such as Walkman, cassette player, CD players, and MP3 players (1). PLD use has become one of the commonest daily habits among teenagers and young adults (2). The World Health Organization (WHO) estimates that 1.1 billion young people under 35 years could be at risk of NIHL from unsafe listening practices while using PLDs (3). The output of most PLDs often exceeds safe levels $(80 \mathrm{~dB})$ and with prolonged use, it can be considered a health hazard with the later development of NIHL $(4,5)$. Most PLD users are unaware that they are putting themselves at risk of sensorineural hearing loss (6). High-intensity sounds can cause permanent hearing loss at chronic exposures of an average $85 \mathrm{~dB}$ or higher (7). The cochlea contains a limited number of hair cells that, when damaged by noise exposure, they cannot regenerate, resulting in permanent hearing loss $(8,9)$. It has been found that extended high frequency regions of the cochlea are affected first by acoustic trauma. Both the intensity and duration of noise exposure determine the potential for damage to the hair cells of the inner ear (10). There has been much discussion among researchers regarding the hazards of recreational noise on hearing acuity during adolescence, however, no standard guideline has been presented. Thus, the rationale of our study is to generate an evidence-based protocol to assess and early discover subtle changes in hearing sensitivity after exposure to recreational noise in order to protect the hearing of PAD users.

\section{Material and Methods \\ 2.1. Participants and inclusion criteria}

This case-control study was conducted on young adults, accompanied with a relative, seeking hearing assessment in the Audiology Unit, Sohag University Hospital, from March 2018 to Jan 2019. We selected 82 young adults as Group I (case group) with the following inclusion criteria: age range from 15 to 20 years, regular use of PADs in the previous 6 months, no hearing complaint, no history of hearing loss, exposure to noise, ototoxic drugs or ear operation. Group II (control group) consisted of 35 young adults with the following inclusion criteria: age from 15 to 20 years, never used PADs, no hearing complaint, no history of hearing loss, no exposure to noise, ototoxic drugs or ear operation. All participants had free otoscopic examination and tympanometry. Regarding the sample size calculation, the 15-20 years age group in Egypt amounts to 17 million, so, the accepted number of cases using PLDs in this age group was estimated to be 62 . However, we selected a larger number of cases. The number of controls could be the same as cases or $40 \%$ of them so we selected 35 controls.

\subsection{Questionnaire and instruments}

This was conducted only for the case group. It is a self-administered questionnaire which was used to collect data from the case participants on the PAD device: (type of device, duration of device use, average time of PAD: days per week and hours per day). Participants were also asked about the preferred listening volume of PADs with 5 options 1 (Very Loud), 2 (Somewhat loud), 3 (Loud), 4 (Medium) and 5 (Low Volume). Participants were asked if they experienced any problems immediately after PAD use (tinnitus, hearing loss, earache or irritability). Pure tone audiometry was conducted on the participants in the two groups. It was carried out inside a sound treated room using an audiometer (Interacoustics AD629). The hearing of the all participants was screened at first at frequencies between $0.25 \mathrm{kHz}$ and $8 \mathrm{kHz}$ at $20 \mathrm{~dB}$ HL to ensure they had no degree of hearing loss. Extended high frequency (EHF) audiometry was carried out on all participants at frequencies between 10 and $16 \mathrm{kHz}$ using headphones for EHF (HDA 200). The maximum output level of the audiometer was taken as the hearing threshold if the participant failed to detect the loudest tone produced by the audiometer. Immitencemetry (Interacoustics AT235) was carried out on all participants to ensure normal middle-ear pressure prior to Otoacoustic Emission (OAE) testing. TEAOE was measured on the all participants using a diagnostic OAE analyzer (Intelligent Hearing Systems, Smart OAE) connected to a portable computer running the Smart OAE software. The participant was seated comfortably in a sound-attenuated booth with limitation of his movements. The probe (Etymotic research, ER-10D) was placed in the external ear canal of the participant with the appropriate-sized ear tip; the fitting was confirmed by the software with in-the-ear calibration. The stimulus is 75 ls clicks (rectangular pulses) with peak equivalent level of $80 \mathrm{~dB}$ SPL at the rate of $20 \mathrm{~s}-1$. The number of averages was 1024 , the filter was set at $0.5-5 \mathrm{kHz}$ and the time window was 25 minutes with the first 2.5 minutes blanked out. The overall amplitude of the Transient Evoked Otoacoustic Emissions (TEOAE) spectrum and TEOAE amplitudes at four frequency bands $(1,2,3$ and $4 \mathrm{kHz})$ were determined by the software. The software's artefact rejection setting excluded the unwanted signals. The software also measured the noise floor during the recordings and calculated the corresponding signal-to-noise ratio (SNR) for the emissions. The pass response was considered if the response at a particular frequency pair or frequency band was greater than the noise floor (SNR $0 \mathrm{~dB}$ ). 


\subsection{Research ethics}

Written informed consent was granted by all the participants after explanation of the reasons for conducting the study. Ethical approval for conducting the study was granted by the Ethics Committee, Medical College. The work was carried out in accordance with the code of Ethics of the World Medical Association (Declaration of Helsinki) for experiments involving humans and was approved by the Ethical Committee of the Faculty of Medicine, Sohag University, Egypt (Ref.: 23/2019). The case group was instructed to refrain from listening to their devices for 24 hours prior to the tests.

\subsection{Statistical analysis}

Data were analyzed using the Statistical Package for the Social Sciences (SPSS) software (version 23). Statistical methods used included descriptive analysis (mean, range and standard deviation) significance level in examining the hypotheses was p-value of less than 0.05 .

\section{Results}

\subsection{General findings}

As regards the gender distribution, there were $50(60.98 \%)$ males and $32(39.02 \%)$ females. In the control group, the number of the participants was 35 with the age range from 15-20 years and the mean age was $16.82 \pm 1.21 \mathrm{SD}$. As regards the gender distribution, there were $22(62.86 \%)$ males and $13(37.14 \%)$ females. Smartphones, for listening to music, were the sole PAD of choice by the entire case group. As regards to the type of earphones, insert phones were being used by $63(76.83 \%)$ and headphones were being used by $19(23.17 \%)$. The duration and frequency of PAD usage is shown in Table 1. When we study the most important problems that occurred immediately after PAD use, tinnitus was first, followed by hearing loss, irritability and finally, earache (Table 2).

Table 1. PAD use characteristics in the case group:

\begin{tabular}{|l|l|l|l|l|}
\hline \multicolumn{2}{|l|}{ Variable } & Males, $\mathrm{n}(\%)$ & Females, $\mathrm{n}(\%)$ & Total, $\mathrm{n}(\%)$ \\
\hline Duration of PAD usage (year) & $<1$ & $13(26)$ & $9(28.13)$ & $22(26.83)$ \\
& $1-3$ & $21(42)$ & $14(43.75)$ & $35(42.68)$ \\
& $>3$ & $16(32)$ & $9(28.13)$ & $25(30.49)$ \\
\hline Days of PAD use/week & $0-3$ days & $17(34)$ & $9(28.13)$ & $26(31.71)$ \\
& $\geq 4-7$ days & $33(66)$ & $23(71.87)$ & $56(68.29)$ \\
\hline Hours of PAD use/day & $\leq 1$ hour & $17(34)$ & $13(40.63)$ & $30(36.59)$ \\
& $\geq 1$ hours & $33(66)$ & $19(59.37)$ & $52(63.41)$ \\
\hline PAD Volume preference & Very Loud & $19(38)$ & $13(40.63)$ & $32(39.02)$ \\
& Somewhat Loud & $14(28)$ & $9(28.13)$ & $23(28.05)$ \\
& Loud & $12(24)$ & $6(18.75)$ & $18(21.95)$ \\
& Medium & $5(10)$ & $3(9.37)$ & $8(9.76)$ \\
& Low & $0(0)$ & $1(3.12)$ & $1(1.22)$ \\
\hline
\end{tabular}

Table 2. Problems immediately occurs after PAD use:

\begin{tabular}{|l|l|l|l|l|}
\hline Questionnaire data & Males, $\mathrm{n}(\%)$ & Females, $\mathrm{n}(\%)$ & Total, $\mathrm{n}(\%)$ \\
\hline Tinnitus & Very likely & $22(44)$ & $15(46.88)$ & $37(45.12)$ \\
& Quiet/Somewhat/Likely & $15(30)$ & $10(31.25)$ & $25(30.49)$ \\
& Unlikely & $13(26)$ & $7(21.87)$ & $20(24.39)$ \\
\hline Hearing loss & Very likely & $11(22)$ & $5(15.63)$ & $16(19.51)$ \\
& Quite/Somewhat/Likely & $29(58)$ & $21(65.63)$ & $50(60.98)$ \\
& Unlikely & $10(20)$ & $6(18.74)$ & $16(19.51)$ \\
\hline Irritability & Very likely & $9(18)$ & $5(15.63)$ & $14(17.07)$ \\
& Quite/Somewhat/Likely & $24(48)$ & $18(56.25)$ & $42(51.23)$ \\
& Unlikely & $17(34)$ & $9(28.12)$ & $26(31.70)$ \\
\hline Earache & Very likely & $5(10)$ & $4(12.5)$ & $9(10.98)$ \\
& Quite/Somewhat/Likely & $23(46)$ & $19(59.38)$ & $42(51.22)$ \\
& Unlikely & $22(44)$ & $9(28.12)$ & $31(37.80)$ \\
\hline
\end{tabular}

\subsection{Pure tone audiometry and EHF audiometry}

At frequencies between $0.25 \mathrm{kHz}$ and $8 \mathrm{kHz}$, all participants were shown to be within normal hearing sensitivity with the mean threshold (19.44 $\pm 4.41 \mathrm{SD}$ for the right ear and 19.61 $\pm 4.50 \mathrm{SD}$ for the left ear). The odds ratio between case and control groups was 165.00 with a high degree of significance $(\mathrm{p}=0.0004)$. The whole case group 
showed hearing threshold shift at all frequencies from 10 to $16 \mathrm{KHz}$ for both ears (Table 3). The largest hearing threshold shift occurred in the case group that used PADs for two or more years. In the current study, we found that the greater hearing threshold shift occurred with the PAD group who used it for three or more years mainly in 14 and $16 \mathrm{KHz}($ Tables 4, 5)

Table 3. The mean \& SD of EHF threshold for the right \& left ears in both groups

\begin{tabular}{|c|c|c|c|c|c|c|c|c|c|}
\hline \multirow{3}{*}{$\begin{array}{l}\text { Frequency } \\
(\mathrm{kHz})\end{array}$} & \multicolumn{4}{|l|}{ Mean } & \multicolumn{4}{|l|}{ SD } & \multirow{3}{*}{ p-value } \\
\hline & \multicolumn{2}{|c|}{ Control group } & \multicolumn{2}{|c|}{ Case group } & \multicolumn{2}{|c|}{ Control group } & \multicolumn{2}{|c|}{ Case group } & \\
\hline & Right ear & Left ear & Right ear & Left ear & Right ear & Left ear & Right ear & Left ear & \\
\hline 10 & 13.25 & 13.33 & 20.97 & 21.01 & 4.86 & 4.90 & 11.02 & 11.08 & $0.0001 *$ \\
\hline 12 & 25.42 & 25.62 & 35.52 & 35.49 & 8.94 & 8.92 & 18.27 & 18.30 & $0.0004 *$ \\
\hline 14 & 31.22 & 31.17 & 39.35 & 39.41 & 10.45 & 10.50 & 18.02 & 18.08 & $0.0043^{*}$ \\
\hline 16 & 49.11 & 49.19 & 61.78 & 61.81 & 17.52 & 17.48 & 21.25 & 21.31 & $0.0005 *$ \\
\hline
\end{tabular}

* There was a highly statistically significant difference in the threshold of EHF in the Control Right ear vs. Case Right ear \& between Control left ear vs. Case left ear.

Table 4. The effect of duration of exposure on the mean \& SD of EHF thresholds in the case group

\begin{tabular}{|l|l|l|l|l|l|}
\hline \multirow{2}{*}{ Frequency $(\mathrm{kHz})$} & \multicolumn{2}{|l|}{$<$ year } & \multicolumn{2}{|c|}{$>3$ years } & \multirow{2}{*}{ p-value } \\
\cline { 2 - 5 } & Mean & SD & Mean & SD & \\
\hline 10 & 20.2 & 11.02 & 23.01 & 12.10 & 0.1220 \\
\hline 12 & 32 & 16.87 & 39.24 & 19.92 & $0.0130^{*}$ \\
\hline 14 & 35.21 & 18.79 & 43.25 & 19.88 & $0.0086^{*}$ \\
\hline 16 & 55.35 & 22.23 & 65.21 & 23.35 & $0.0063^{*}$ \\
\hline
\end{tabular}

* There was a statistically significant difference in the threshold of EHF in the Case group with increasing year of exposure, mainly in 14 \& $16 \mathrm{KHz}$.

Table 5. The correlation between different variables and EHF threshold in the case group

\begin{tabular}{|l|l|l|}
\hline Variable & $\mathrm{r}$ & $\mathrm{p}$-value \\
\hline Years of exposure & 0.933 & $0.00001^{*}$ \\
\hline Days of exposure & 0.89 & $0.00001^{*}$ \\
\hline Hours of exposure & 0.845 & $0.00001^{*}$ \\
\hline Volume of PLDs & 0.957 & $0.00001^{*}$ \\
\hline
\end{tabular}

* Strong positive correlation between different variables \& EHF threshold.

\subsection{Results of $\mathrm{OAE}$}

The TEOAE responses were present in all participants of the control group, while in the case group five participants $(6.09 \%)$ showed no responses; three of whom had bilateral absence of response. The odds ratio for TEOAE response was 1.0649 with no statistically significant differences between case and control groups $(p=0.8264)$. As regards the TEOAE amplitude, the mean amplitude of the overall TEOAE spectrum was lower in the case group compared with the controls. In the right ear, it was $16.85 \pm 3.02 \mathrm{~dB}$ in the case versus $18.92 \pm 3.14 \mathrm{~dB}$ in the control with the degree of the significance less than 0.01 . In the left ear, it was $16.92 \pm 4.28 \mathrm{~dB}$ in the case versus $19.01 \pm 3.76$ in the controls with the degree of the significance less than 0.05 .

\section{Discussion}

The current study was conducted to investigate the patterns of PAD use in young adults and the risk of exposure to recreational noise, to consider the magnitude of the problem and the methods applied to early identify it. The participants selected were young adults with the age range from 15-20 years. In the case group, there was male predominance with a percentage of $60.98 \%$. This was consistent with a study done by Saurav et al. who found that male students were more likely to listen to PADs with greater frequency than female students (11). This can be considered a very dated view in most cultures worldwide. Women are just as likely to be walking, running or riding bicycles. Our findings regarding the average duration of PAD use is in agreement with a cross sectional study done among Iranian adolescent students, which concluded that 33 of them listened to PADs for more than two hours per day (12). Similarly, another study was conducted at a medical college in India and reported that the majority of the students (77.7\%) used PADs more than one hour per day (13). However, in the study done by Saurav et al., they concluded that the majority (70\%) of the participants were using PADs for less than one hour per day while the rest 
were using PADs for two or more hours (11). As regards the PAD volume preference, the largest percentage $(39.02 \%)$ prefer very loud volume (Table 1). This is in agreement the with study done by Saurav et al. in which they found the preferred mode of listening was very loud in $20 \%$ of the case participants (11).

Regarding the most important problems that occurred immediately after PAD use, our findings are similar to a previous study done among US college students in California, which found that tinnitus and hearing loss were observed in $15.9 \%$ and $11.2 \%$ of the participants respectively (14). This can be explained by the fact that exposure to high and continuous noise can lead to ear affection first (hearing loss \& tinnitus) followed by neurological complaints (irritability). In the current study, conventional pure tone audiometry $(0.25$ to $8 \mathrm{KHz})$ showed no abnormality among the case group at all frequencies, which is in agreement with Jian et al. who found that the hearing thresholds of the PAD subgroup were significantly higher than those of the control group in the range of 10 to $20 \mathrm{kHz}$, although they had completely normal hearing thresholds at 0.5 to $8 \mathrm{kHz}$ (15). In another study, the mean hearing thresholds were found to be significantly higher at high frequencies $(8-12 \mathrm{KHz})$ in PLD users compared to control group and more threshold shift was observed in the subgroup who listened for $7 \mathrm{~h} /$ week compared with those who listened for 2-7 h/week (16). Young adult PLD users who listened to their devices for $1 \mathrm{~h} /$ day had normal thresholds at conventional frequencies but showed elevated thresholds at EHFs (17). This means that the long-term exposure to loud sounds from PADs can appear in the high frequency region even if the conventional PTA appeared within the normal frequency region. Thus, EHF audiometry is more sensitive than conventional frequency audiometry in early detection of NIHL.

The duration of noise exposure is an important factor for NIHL. We found that the greater hearing threshold shift occurred with the PAD group who used it for three or more years mainly in 14 and $16 \mathrm{KHz}$. This is in agreement with the study done by Jian et al. who concluded that the hearing threshold shift occurred in a broad frequency range and had a high incidence of hearing damage in the subgroup that used PADs for 5 years (15). This revealed that the risk of hearing impairment is increased, the longer the duration of noise exposure (Table 5). The generation of evoked OAEs is attributed to the active outer hair cell (OHC) function (18). In the current study, five participants (6.09\%) showed no responses in TEOAE; three of whom had bilateral absence of response. As regards the TEOAE amplitude, it was lower in PLD users than in those of the control group, which indicates an initial damage of their OHCs. In other studies, the occurrence and amplitude of TEOAEs were found to be decreased in regular young PLD users compared with non-users $(19,20)$. Compared with audiometry, early cochlear damage due to PLD usage may be more readily detected using OAE measurements $(20,21)$.

\section{Strength and limitation of the study}

The strengths of this study were the use of both subjective and objective methods for assessing the hazards of PLDs on the users. However, there were study limitations such as the number of participants, which requires a larger population when in order to generalize our findings. Also, there were limitations at various stages of the research including the fact that some participants failed to complete the whole test, and thus were replaced.

\section{Conclusions}

The long-term use of PLDs can induce NIHL. This could translate into a global hearing health problem. A standard screening protocol consisting of a questionnaire, EHF audiometry and TEOAE should be performed routinely for PLD users who use the devices for 3 years or more. Our study recommends preventive tools such as educational lectures, posters and campaigns directed at PLD users in order to educate them regarding the safe mode for listening to PLDs by decreasing the duration and intensity of use. In addition, it would be beneficial that the audiologist perform a screening protocol consisting of a questionnaire, EHF audiometry and TEOAE to early identify NIHL in PLDs users.

\section{Acknowledgments:}

Many thanks to the staff of the Audio-vestibular Unit, Sohag University Hospital, Sohag, Egypt, for their help and support in this original article.

\section{Conflict of Interest:}

There is no conflict of interest to be declared. 


\section{References:}

1) Williams, W. (2005). Noise exposure levels from personal stereo use. International Journal of Audiology, 44(4), 231-236. doi: 10.1080/14992020500057673 PMid: 16011051

2) Serra MR, Biassoni EC, Richter U. Recreational noise exposure and its effects on the hearing of adolescents. Part 1: an interdisciplinary long-term study. Int 1 Audiol 2005; 44:65-73. doi: 10.1080/14992020400030010 PMid: 15913154

3) WHO, 2015 Hearing loss due to recreational exposure to loud sounds: A review. Geneva: World Health Organization; 2015.

4) Fligor BJ, Cox LC. Output levels of commercially available Personal compact disc players and the potential risk to hearing. Ear Hear. 2004;25:513-27. doi: 10.1097/00003446-200412000-00001 PMid: 15604913

5) Williams W. Noise exposure levels from Personal stereo use. Int J Audiol. 2005;44:231-6. doi: 10.1080/14992020500057673 PMid: 16011051

6) Bethesda 1. Noise-Induced Hearing Loss. National Institute on Deafness and Other Communication Disorders. 2007; 97-4233-9.

7) Crandell C, Mills TL, Gauthier R. Knowledge, behaviors and attitudes about hearing loss and hearing protection among racial/ethnically diverse young adults. J Natl Med Assoc 2004; 96(2):17686.

8) Daniel E. Noise and hearing loss: a review. J Sch Health 2007;77: 225-31. doi: 10.1111/j.17461561.2007.00197.x PMid: 17430434

9) Harrison RV. Noise-induced hearing loss in children: A "less than silent" environmental danger. Peadiatr Child Health 2008; 13: 377-82. doi: 10.1093/pch/13.5.377 PMid: 19412364 PMCid: PMC2532893

10) El Dib RP, Silva EM, Morais JF, Trevisani VF. Prevalence of high frequency hearing loss consistent with noise exposure among people working with sound systems and general population in Brazil: A crosssectional study. BMC Public Health 2008; 8:151. doi: 10.1186/1471-2458-8-151 PMid: 18462490 PMCid: PMC2409328

11) Saurav, B., Suneela, G., Meghachandra, S., Charu, K.\& Maulana, A.: Personal audio devices use patterns associated with risks of hearing loss and compromised road safety among medical students in Delhi 12th ICBEN Congress on Noise as a Public Health Problem 2017.

12) Ansari H, Mohammadpoorasl A, Rostami F, Maleki A, Sahebihagh MH, Naieni KH. Pattern of Use of Earphone and Music Player Devices among Iranian Adolescents. International Journal of Preventive Medicine. 2014; 5(6):776-81.

13) Rekha T, Mithra P, Bukelo M, Unnikrishnan B, Kumar N, Ballala K. Perceptions and practices regarding use of personal listening devices among medical students in coastal South India. Noise Heal. 2011 SepOct;13(54):329-32. doi: 10.4103/1463-1741.85500 PMid: 21959112

14) Hoover A, Krishnamurti S. Survey of college students' MP3 listening: Habits, safety issues, attitudes, and education. Am J Audiol 2010; 19:73-83. doi: 10.1044/1059-0889(2010/08-0036)

15) Jian-Hua, P., Ze-Zhang, T. \& Zhi-Wu, H.: Risk of Damage to Hearing from Personal Listening Devices in Young Adults. The Journal of Otolaryngology 2007; 36: 181-5. doi: 10.2310/7070.2007.0032

16) Meyer-Bisch $C$ Epidemiological evaluation of hearing damage related to strongly amplified music (personal cassette players, discotheques, rock concerts)-high-definition audiometric survey on 1364 subjects. Audiology 1996; 35(3):121-42. doi: 10.3109/00206099609071936 PMid: 8864255

17) Peng JH, Tao ZZ, Huang ZW Risk of damage to hearing from personal listening devices in young adults. J Otolaryngol 2007; 36(3):181-5. doi: 10.2310/7070.2007.0032

18) Kemp DT Otoacoustic emissions, their origin in cochlear function, and use. Br Med Bull 2002; 63:223-41. doi: 10.1093/bmb/63.1.223 PMid: 12324396

19) LePage E. \& Murray N. Latent cochlear damage in personal stereo users: a study based on click-evoked otoacoustic emissions. Med J Aust 1998; 169(11-12):588-92. doi: 10.5694/j.1326-5377.1998.tb123426.x PMid: 9887901

20) Montoya FS, Ibarguen AM, Vences AR, del Rey AS, Fernandez JM Evaluation of cochlear function in normal-hearing young adults exposed to MP3 player noise by analyzing transient evoked otoacoustic emissions and distortion products. J Otolaryngol Head Neck Surg 2008; 37(5):718-24

21) Bhagat SP, Davis AM Modification of otoacoustic emissions following ear-level exposure to MP3 player music. Int J Audiol 2008; 47(12):751-60. doi: 10.1080/14992020802310879 PMid: 19085399 